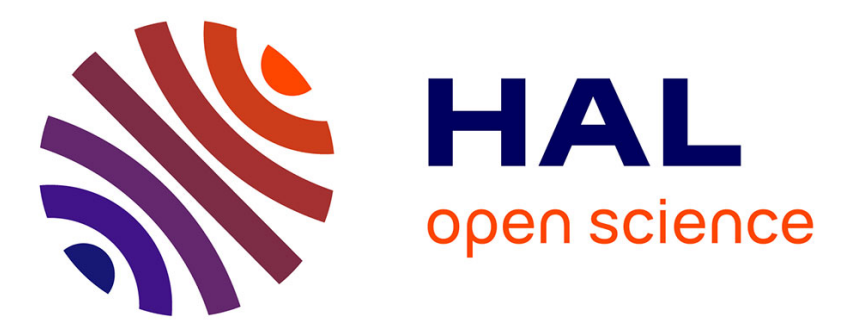

\title{
The Offset to an Algebraic Curve and an Application to Conics
}

François Anton, Ioannis Z. Emiris, Bernard Mourrain, Monique Teillaud

\section{To cite this version:}

François Anton, Ioannis Z. Emiris, Bernard Mourrain, Monique Teillaud. The Offset to an Algebraic Curve and an Application to Conics. International Conference on Computational Science and its Applications, May 2005, Singapore, Singapore. pp.683-696. inria-00350878

\section{HAL Id: inria-00350878 \\ https://hal.inria.fr/inria-00350878}

Submitted on 7 Jan 2009

HAL is a multi-disciplinary open access archive for the deposit and dissemination of scientific research documents, whether they are published or not. The documents may come from teaching and research institutions in France or abroad, or from public or private research centers.
L'archive ouverte pluridisciplinaire HAL, est destinée au dépôt et à la diffusion de documents scientifiques de niveau recherche, publiés ou non, émanant des établissements d'enseignement et de recherche français ou étrangers, des laboratoires publics ou privés. 


\title{
The Offset to an Algebraic Curve and an Application to Conics
}

\author{
François Anton ${ }^{1}$, Ioannis Emiris ${ }^{2}$, \\ Bernard Mourrain ${ }^{3}$, and Monique Teillaud ${ }^{3}$ \\ 1 Department of Computer Science, \\ University of Calgary, 2500 University Drive N.W., \\ Calgary, Alberta, T2N 1N4, Canada \\ antonf@cpsc.ucalgary.ca \\ 2 Department of Informatics and Telecommunications, \\ National Kapodistrian University of Athens, \\ Panepistimiopolis 15784, Greece \\ 3 INRIA Sophia-Antipolis, B.P. 93, 2004, \\ route des Lucioles, 06902, Sophia-Antipolis, France
}

\begin{abstract}
Curve offsets are important objects in computer-aided design. We study the algebraic properties of the offset to an algebraic curve, thus obtaining a general formula for its degree. This is applied to computing the degree of the offset to conics. We also compute an implicit equation of the generalised offset to a conic by using sparse resultants and the knowledge of the degree of the implicit equation.
\end{abstract}

\section{Introduction}

An important object in nonlinear computational geometry, Computer-aided Geometric Design and geometric modelling is the offset of a given curve or surface, which is defined as the locus of points at a given distance. A related question concerns the bisector of two curves or surfaces. Both problems have been addressed for some classes of curves or surfaces, mostly for their applications (see [1] for the application to the Voronoi diagram of semi-algebraic sets).

What Hoffmann and Vermeer [8] define as offset curves, Arrondo, Sendra and Sendra 2] define as generalised offset curves. The extraneous solutions (corresponding to a point of the curve or surface which corresponds to infinitely many points on the generalised offset) have been addressed in [8]. Hoffmann and Vermeer [8] did not address the offset computations, but they gave some numerical examples computed using Gröbner bases. Arrondo, Sendra and Sendra [2] computed the genus of the generalised offset curve.

In this paper, we will address the degree of the offset to an algebraic plane curve in its most general setting. Our main contributions are a general formula for the degree of the offset curve and its application for the determination of an implicit equation of the generalised offset to a conic. The conic is defined implicitly by a formal polynomial (i.e., a polynomial whose coefficients are formal 
constants). We used the general formula of the degree of the offset curve to eliminate the extraneous factors from the sparse resultant [3, 4] in order to get an implicit equation defining the generalised offset to a conic.

This paper is organised as follows: in Section 2, we study the equation of the offset. In Section 3, we study the algebraic properties of the offset to an algebraic curve in order to determine its degree. In Section 4, we show how to compute the implicit equation of the generalised offset to a conic.

\section{Equations Defining the Offset}

This section uses notions of algebraic geometry; for further background one may consult [9]. We assume the most general setting for the problem at hand, i.e. complex numbers and we let $k=\mathbb{C}$. In this paper, we assume that $V\left(f_{1}, \ldots, f_{s}\right) \subset E$ denotes the set of all the points of $E$ whose coordinates $\left(x_{1}, \ldots, x_{n}\right)$ satisfy the polynomial equations $f_{1}\left(x_{1}, \ldots, x_{n}\right)=\cdots=f_{s}\left(x_{1}, \ldots, x_{n}\right)=0$. If $E=k^{n}$ is an affine space, then the set $V$ is called an affine variety. If $E=\mathbb{P}^{n}$ is a projective space over $k$, then the set $V$ is called a projective variety. Distances are always measured in the Euclidean metric. The distance of a point to a curve is the smallest distance of the point from any point on the curve.

Definition 1. (Offset curve) Let $C=V(f) \subset k^{2}$, for $f \in k[x, y]$, be an algebraic curve and $R \in \mathbb{R}^{+}$be the offset parameter. The $R$-offset curve to $C$ is the locus of points lying at distance $R$ from $C$ (see Figure 1).

Definition 1 is equivalent to saying that each point $q=(u, v)$ of the offset curve is the centre of a circle $\mathcal{D}$ of radius $R$, which is tangent to $C$, and does not contain any point of $C$ in its interior. We will suppose that $f$ has positive degree (thus $C$ is not empty) and $R \neq 0$ unless stated otherwise.

Definition 2. (Generalised offset) The generalised $R$-offset curve to $C$ denoted $\mathcal{O}$ is the locus of the centres $q=(u, v)$ of circles $\mathcal{D}$ of radius $R$, that are tangent to $C$ (see Figure 1).

The $R$-generalised offset to $C$ is a superset of the true $R$-offset curve to $C$. Indeed, the circle centred on a point $q^{\prime}$ of the true $R$-offset curve to $C$ of radius
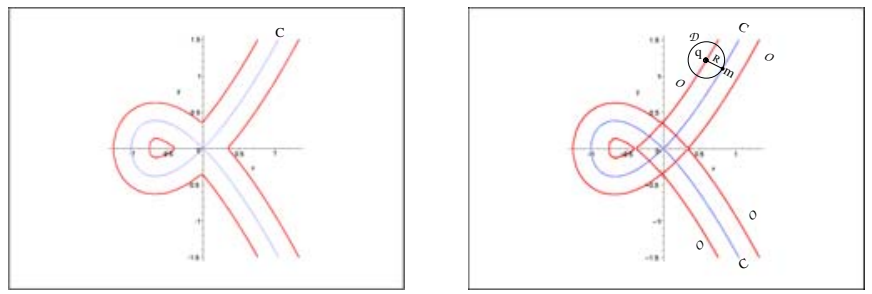

Fig. 1. The Maple plots of a strophoid (C), its true offset (left) and of its generalised offset $\mathrm{O}$ (right) 
$R$ is tangent to $C$. Its interior does not intersect $C$. The Zariski closure [9] of an affine (resp. projective) variety $V$ is the smallest affine (resp. projective) variety containing $X$. The generalised offset to a hypersurface $\nu$ at distance $R$ is the Zariski closure of the set of intersection points of the spheres with centre on $\nu$ and radius $R$, and the normal lines to $\nu$ at the centre of the spheres, cf. 2. We will now establish the systems of equations and inequalities that define the generalised $R$-offset $\mathcal{O}$ and the true $R$-offset to an algebraic curve $C=V(f)$. The normal to $C$ at a given point $p=(x, y) \in C$ is the set of points $q=(u, v)$ for which $n_{x, y}(u, v)=0$, where $n_{x, y}(u, v)=-f_{y}(x, y) \cdot(u-x)+f_{x}(x, y) \cdot(v-y)$. Here $f_{x}$ and $f_{y}$ denote the partial derivatives of $f$. A point $p$ of an algebraic curve $V(f) \subset k^{2}$ is called a singular point if, and only if, both partial derivatives of $f$ vanish at $p$ (i.e., $f_{x}(p)=f_{y}(p)=0$ ). For a given $q=(u, v) \in$ $k^{2}$, let $\mathcal{M} \subset \mathbb{R}^{2}$ be the the set of points $m=(\alpha, \beta) \in C \cap \mathbb{R}^{2}$ such that $n_{\alpha, \beta}(u, v)=0$. This condition is achieved whenever the normal to $C \cap \mathbb{R}^{2}$ at $m$ passes through $q$, or $m$ is singular. In the general case, $\mathcal{M}$ is finite. However, if $C \cap \mathbb{R}^{2}$ is a circle centred on $q$, then $\mathcal{M}=C \cap \mathbb{R}^{2}$. More generally, if $f$ is not square-free, there is an infinity of singular points. To get in all cases a finite set of points $m$ of $C \cap \mathbb{R}^{2}$ such that $n_{\alpha, \beta}(u, v)=0$, we use $\mathcal{S}=\mathcal{M}$ when $\mathcal{M}$ is finite, and $\mathcal{S}=\{w\}$ for an arbitrary point $w$ of $C \cap \mathbb{R}^{2}$ when $\mathcal{M}$ is infinite.

Lemma 1. The set of all the closest points on $C \cap \mathbb{R}^{2}$ from $q$ is contained in $\mathcal{M}$.

Proof. The polynomial $n$ (considered here as a polynomial in $\alpha, \beta$ for fixed $u, v$ ) defining $\mathcal{M}$ expresses half of the differential of the scalar product $\overrightarrow{q m} \cdot \overrightarrow{q m}$ (which is equal to the square of the Euclidean distance $\delta(q, m)$ ) with respect to $m$. The closest points on $C \cap \mathbb{R}^{2}$ from $q$ are global minima of the Euclidean distance $\delta(q, m)$, so, the differential (and thus, $n$ ) vanishes on them.

The point $q=(u, v)$ on the generalised $R$-offset curve $\mathcal{O}$ can be constructed from a non-singular point $p=(x, y)$ on $C$ as the intersection of the normal to $C$ at $p$ (given by $n_{x, y}$, as mentioned previously) and the circle $\mathcal{D}$ centred on $p$, and of radius $R$. The equation of this circle is $d_{x, y}(u, v)=0$, where $d_{x, y}(u, v)=$

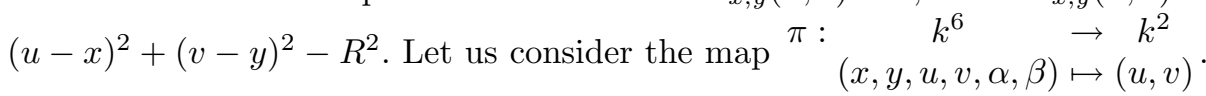
$\pi$ can also be seen as the canonical projection of $k^{4} \subset k^{6}$ onto $k^{2}$. If we consider the inclusion map from $k^{2}$ to $k^{4}, V(f)$ can be seen as a variety of $k^{4}$, which is the image of $C$ by this inclusion map. Let $n(x, y, u, v)=n_{x, y}(u, v)$ and $d(x, y, u, v)=d_{x, y}(u, v)$ be considered now as polynomials in $k[x, y, u, v]$. $V(n)$ is a proper subset of $k^{4}$ provided that $p=(x, y)$ is not singular. If $f$ is not square-free, there is an infinity of singular points. From now on, the affine and projective varieties will be considered in different underlying spaces. When necessary, we will specify the underlying space by an inclusion, e.g. $V(f) \subset k^{4}$ or $V(f) \subset k^{2}$. The generalised $R$-offset $\mathcal{O}$ is the image by $\pi$ of the affine variety of $k^{4}$ defined by the following system of equations and inequalities: 


$$
\left\{\begin{array}{l}
f(x, y)=0 \\
n(x, y, u, v)=0 \\
d(x, y, u, v)=0 \\
f_{x}(x, y) \neq 0 \text { or } f_{y}(x, y) \neq 0
\end{array}\right.
$$

$\mathcal{O}$ is the image of $(V(f) \cap V(n) \cap V(d)) \backslash V\left(f_{x}, f_{y}\right)$ by the canonical projection $\pi: k^{4} \rightarrow k^{2}$ onto the $(u, v)$-plane. The true $R$-offset lies in $\mathbb{R}^{2}$ and is obtained as the difference of the generalised $R$-offset $\mathcal{O}$ and the union of the images by $\pi$ of the sets defined by the following system of equations and inequalities, for each point $m=(\alpha, \beta)$ of $\mathcal{S}$ :

$$
\left\{\begin{array}{l}
f(\alpha, \beta)=0 \\
n(\alpha, \beta, u, v)=0 \\
(u-\alpha)^{2}+(v-\beta)^{2}-R^{2}<0
\end{array}\right.
$$

\section{The Degree of the Offset Curve}

This section relies on several algebraic concepts, which can be found in more detail in [9. Consider polynomials in the variables $x, y, u$ and $v$ with coefiicients in $k$ and the projective space $\mathbb{P}^{4}$ (of lines in $k^{5}$ passing through the origin). The homogenisation variable will be denoted by $t$. We consider the point $q=$ $(u, v)$ on the generalised $R$-offset curve $\mathcal{O}$ constructed above from an arbitrary point $p=(x, y)$ on $C$. Let $\bar{g}=t^{\operatorname{degree}(g)} g\left(\frac{x}{t}, \frac{y}{t}, \frac{u}{t}, \frac{v}{t}\right)$ be the homogenisation of a polynomial $g, \bar{V}$ denote the projective closure of the variety $V$, i.e. the smallest projective variety containing $V$, and $g^{T}$ denote the polynomial defining the component at infinity (i.e. the points with homogeneous coordinate equal to zero) of $\overline{V(g)}$. Note that $\overline{V(g)}=V(\bar{g})$-p. 33]. Thus, $V(\bar{f}), V(\bar{n}), V(\bar{d}) \subset \mathbb{P}^{4}$.

We are now going to decompose the projective closure of $V(f, n, d) \subset k^{4}$ into the union of its component at infinity, its singular component (points induced by singular points $p$ on $C$ ), and the generalised offset considered in $k^{4}$ (i.e. the affine variety $\left.V(f, n, d) \backslash V\left(f_{x}, f_{y}\right) \subset k^{4}\right)$. We will thus obtain the generalised offset considered in $k^{4}$ as a difference of projective varieties, and we will determine their dimensions and degrees in order to determine the degree of the generalised offset.

Let us define $W=\overline{V(f)} \cap \overline{V(n)} \cap \overline{V(d)}, W_{a}=W \backslash\left(\overline{V\left(f_{x}, f_{y}\right)} \cup V(t)\right) \subset \mathbb{P}^{4}$, $W_{S}=W \cap \overline{V\left(f_{x}, f_{y}\right)}$, and $W_{\infty}=W \cap V(t)$. We recall here the definition of a quasi-projective variety. In the Zariski topology, the only sets that are closed are algebraic (affine or projective) varieties. A quasi-projective variety is an open subset of a projective variety. $W_{a}$ is a quasi-projective variety since $\overline{V(f)} \cap \overline{V(n)} \cap \overline{V(d)}$ and $\overline{V\left(f_{x}, f_{y}\right)} \cup V(t) \subset \mathbb{P}^{4}$ are projective varieties. $W_{S}$ is a projective variety and $W_{\infty}=V\left(f^{T}, d^{T}, n^{T}, t\right)$ a projective subvariety. A variety $X$ is called reducible if there exist varieties $X_{1} \subset X, X_{2} \subset X, X_{1} \neq X$, $X_{2} \neq X$, such that $X=X_{1} \cup X_{2}$. A polynomial is reducible if it can be factorized into two polynomials of positive degree. Let $d_{1}:=-\iota(u-x)+(v-y), d_{2}:=$ $\iota(u-x)+(v-y)$, and let $\iota$ be a root of the equation $x^{2}+1=0$ in the 
algebraically closed field $k$. Then $V\left(d^{T}\right)=V\left(d_{1}\right) \cup V\left(d_{2}\right)$ is a minimal decomposition, i.e. into the smallest number of irreducible varieties. We consider the ring $k\left[x_{1}, \ldots, x_{n}\right]$ of polynomials in $n$ variables with coefficients in $k$. Let $\left\langle f_{1}, \ldots, f_{s}\right\rangle=\left\{g_{1} f_{1}+\cdots+g_{n} f_{n}: \forall i, g_{i} \in k\left[x_{1}, \ldots, x_{n}\right]\right\}$ denote the ideal generated by $f_{1}, \ldots, f_{s} \in k\left[x_{1}, \ldots, x_{n}\right]$. Let $\sqrt{I}=\left\{f \in k\left[x_{1}, \cdots, x_{n}\right]: \exists n \in \mathbb{N}, f^{n} \in I\right\}$ denote the radical of an ideal $I$. Lastly, for any variety $W$, let $I(W)$ denote the set of all polynomials vanishing on $W$; this is easily shown to be an ideal. For any variety $W=V(p), I(W)=\sqrt{(}\langle p\rangle)$ by Hilbert's Nullstellensatz [9. Now, we will prove some lemmas that will be needed for the proof of Lemma 6. Let $V\left(f^{T}\right)=V\left(h_{1}\right) \cup V\left(h_{2}\right) \cup \cdots \cup V\left(h_{s}\right)$ be minimal: $\forall j \neq i, V\left(h_{i}\right) \not \subset V\left(h_{j}\right)$.

Lemma 2. None of the irreducible components of $V\left(f^{T}\right) \subset \mathbb{P}^{4}$ is contained in an irreducible component of $V\left(d^{T}\right) \subset \mathbb{P}^{4}$. Equivalently, none of the $h_{i}$ divides $d^{T}$.

Proof. Let us first establish the equivalence of the two statements. For any $i$, we have $V\left(h_{i}\right) \subset V\left(d^{T}\right) \Leftrightarrow d^{T} \in I\left(V\left(h_{i}\right)\right)=\sqrt{\left\langle h_{i}\right\rangle}$. Since $h_{i}$ is irreducible, the latter ideal equals $\left\langle h_{i}\right\rangle$. Hence, an equivalent expression is that $h_{i}$ divides $d^{T}$. We will prove the second statement by contradiction. Assume $d^{T} \in I\left(V\left(h_{i}\right)\right) \subset$ $k[x, y, u, v, t]$ for some $i \in\{1,2, \ldots, s\}$. Thus, there exists $g \in k[x, y, u, v, t]$ such that $d^{T}=g \cdot h_{i}$. The sum of the degrees of $g$ and of $h_{i}$ equals 2 . There are three cases, namely that the respective degrees equal 0,2 or 1,1 or 2,0 . If the degree of $h_{i}$ is 2 , then $h_{i}$ is reducible, therefore the first case is not possible. In the second case, $d^{T}=g \cdot(\alpha x+\beta y+\gamma)$, where $\alpha, \beta, \gamma$ are coefficients of $k$. This would imply that the terms in $u^{2}$ and the terms in $v^{2}$ of $d^{T}$ must come from $g$. These terms induce terms in $u^{2} x, u^{2} y, v^{2} x$, and $v^{2} y$ which cannot be canceled. So the second case is also infeasible. In the last case, $h_{i}$ cannot be a constant by definition.

We consider the projective space $\mathbb{P}^{N}$ and we let $\xi \in \mathbb{P}^{N}$ be denoted by $\left(\xi_{0}\right.$ : $\left.\cdots: \xi_{N}\right)$, where $\xi_{i} \in k, i=0, \ldots, N$ and not all the $\xi_{i}$ are 0 . Let $\mathbb{A}_{i}^{N} \subset \mathbb{P}^{N}$ consist of all the points for which $\xi_{i} \neq 0$ (thus, it is isomorphic to the affine space $k^{N}$ ). We now define regular functions, regular mappings of affine varieties, and regular mappings of quasi-projective varieties. Let $X$ be an affine variety in $k^{N}$. A function $g$ on $X$ is regular $[9$ if there exists a polynomial $G$ with coefficients in $k$ such that $g(x)=G(x), \forall x \in X$. Let $Y$ be a variety of $k^{N}$. A mapping $g: X \rightarrow Y$ is regular if there exists $N$ regular functions $g_{1}, \ldots, g_{N}$ on $X$ such that $g(x)=$ $\left(g_{1}(x), . ., g_{N}(x)\right), \forall x \in X$. Let $f: X \rightarrow Y$ be a mapping of quasi-projective varieties and $Y \subset \mathbb{P}^{N}$. This mapping is called regular if for every point $x \in X$ and every open affine set $\mathbb{A}_{i}^{N}$ containing the point $f(x)$ there exists a neighbourhood $U$ of $x$ such that $f(U) \subset \mathbb{A}_{i}^{N}$, and the mapping $f: U \rightarrow \mathbb{A}_{i}^{N}$ is regular. The dimension of a variety $X$ is $\sup \left\{n: X_{0} \varsubsetneqq \cdots \varsubsetneqq X_{n} \subset X\right.$ and $\forall i, X_{i}$ irreducible $\}$.

Theorem 1. 9-theorem.8, Sect.1.6] Let $f: X \rightarrow Y$ be a regular mapping between projective varieties with $f(X)=Y$. Suppose that $Y$ is irreducible and that all the fibres $f^{-1}(y)$ for $y \in Y$ are irreducible and of the same dimension, then $X$ is irreducible. 
Lemma 3. None of the irreducible components of $V\left(f^{T}, d^{T}\right) \subset \mathbb{P}^{4}$ is contained in $V(t) \subset \mathbb{P}^{4}$.

Proof. Notice that $V\left(f^{T}\right)$ and $V\left(h_{i}\right)$ are not contained in the projective hyperplane at infinity $V(t) \subset \mathbb{P}^{4}$. By considering the intersection of two finite set unions, it is clear that the set $V\left(f^{T}, d^{T}\right)$ can be written as the union of all sets $V\left(h_{i}, d_{j}\right)$, for $i=1, \ldots, s, j=1,2$. We shall now show that the $V\left(h_{i}, d_{j}\right)$ are irreducible. Consider the projection $\mathbb{P}^{4} \rightarrow \mathbb{P}^{2}:(t: x: y: u: v) \mapsto$ $(t: x: y)$. It can be restricted to the following sequence of projections:

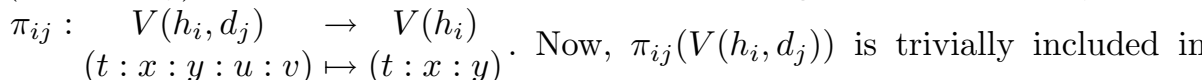
$V\left(h_{i}\right)$. The converse is also true: for any $q=\left(t_{q}: x_{q}: y_{q}\right) \in V\left(h_{i}\right)$, if we consider $q^{\prime}=\left(t_{q}: x_{q}: y_{q}: u: v\right)$ such that $d_{j}(u, v)=0$, then $q^{\prime} \in V\left(h_{i}, d_{j}\right)$ and $\pi_{i j}\left(q^{\prime}\right)=q$. So, $\pi_{i j}\left(V\left(h_{i}, d_{j}\right)\right)=V\left(h_{i}\right)$. The $\pi_{i j}$ are regular mappings of projective varieties. The fibres $\pi_{i j}^{-1}(\omega)$ for $\omega \in V\left(h_{i}\right)$ have dimension 1 since the points on these fibres have fixed $x, y$ and $t$ coordinates, and $u$ and $v$ are related by the equation of $d_{j}$. Thus, all the fibres have the same dimension. Also, each fibre is irreducible since $V\left(d_{j}\right)$ is irreducible. Then, by Theorem 11 we conclude that the $V\left(h_{i}, d_{j}\right)$ are irreducible. We will show that none of these $V\left(h_{i}, d_{j}\right)$ is contained in an irreducible component of $V(t)$. Let us suppose $t \in I\left(V\left(h_{i}, d_{j}\right)\right)$ for some $i \in\{1,2, \ldots, s\}$ and $j \in\{1,2\}$. By Hilbert's Nullstellensatz, $I\left(V\left(h_{i}, d_{j}\right)\right)=\sqrt{\left\langle h_{i}, d_{j}\right\rangle}$. Since the $V\left(h_{i}, d_{j}\right)$ are irreducible, $\sqrt{\left\langle h_{i}, d_{j}\right\rangle}=\left\langle h_{i}, d_{j}\right\rangle$. Then there exists $a, b \in k[x, y, u, v, t]$ such that $t=a h_{i}+b d_{j}$. Since $h_{i}$ and $d_{j}$ don't have monomials with $t$ nor constant terms, $t$ in $a h_{i}+b d_{j}$ must come from $a$ or $b$ or both. Since $h_{i}$ and $d_{j}$ don't have constant terms, the monomial of least total degree containing $t$ in $a h_{i}+b d_{j}$ must have a degree greater than or equal to 1 in the other variables.

Lemma 4. None of the irreducible components of $V\left(f^{T}, d^{T}, t\right) \subset \mathbb{P}^{4}$ is contained in an irreducible component of $V\left(n^{T}\right) \subset \mathbb{P}^{4}$ (or, equivalently, $n^{T}$ does not vanish identically on any irreducible component of $\left.V\left(f^{T}, d^{T}, t\right)\right)$ if, and only if, $f_{x}^{T}+$ $\iota f_{y}^{T} \notin\left\langle h_{i}\right\rangle$ and $f_{x}^{T}-\iota f_{y}^{T} \notin\left\langle h_{i}\right\rangle$ for $i=1,2, \ldots, s$.

Proof. The fact that the statement in parenthesis is indeed equivalent can be proved in a manner analogous to that in the proof of Lemma 2. Now, consider the projection $V\left(h_{i}, d_{j}, t\right) \rightarrow V\left(h_{i}, t\right):(t: x: y: u: v) \mapsto(t: x: y)$, which can be restricted to the following mappings, for $i=1, \ldots, s, j=1,2$ :

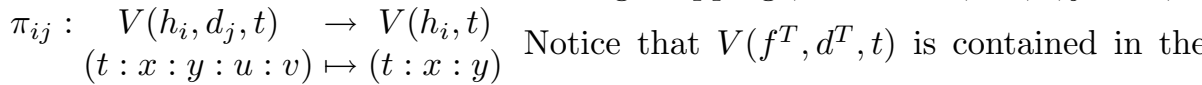
hyperplane at infinity $V(t)$, and so does $V\left(h_{i}, d_{j}, t\right)$. These mappings are regular mappings of projective varieties and $\pi_{i j}\left(V\left(h_{i}, d_{j}, t\right)\right)=V\left(h_{i}, t\right)$. Each fibre of these mappings is irreducible, and $V\left(h_{i}, t\right)$ is also irreducible. The fibres $\pi_{i j}^{-1}(\omega)$ have dimension 1 since the points on these fibres have fixed $x, y, t$ coordinates and their $v$ coordinate is related to $u$ by $d_{j}$, and is therefore also fixed. Thus, all the fibres have the same dimension. Then, we can apply Theorem 1 and conclude that the $V\left(h_{i}, d_{j}, t\right)$ are irreducible, as in the proof of Lemma 3. We will prove 
the first direction. Let us suppose $f_{x}^{T}+\iota f_{y}^{T} \in\left\langle h_{i}\right\rangle$ for some $i \in\{1,2, \ldots, s\}$. We have to show that $n^{T}=0$ on $V\left(h_{i}, d_{1}, t\right)$. Since $\left(f_{x}^{T}\right)+\iota\left(f_{y}^{T}\right) \in\left\langle h_{i}\right\rangle$, there exists a polynomial $g$ such that $\left(f_{x}^{T}\right)+\iota\left(f_{y}^{T}\right)=g \cdot h_{i}$. But, $h_{i}=0$ on $V\left(h_{i}, d_{1}, t\right)$. Thus, $\left(f_{x}^{T}\right)+\iota\left(f_{y}^{T}\right)=0$, i.e. $f_{y}^{T}=\iota f_{x}^{T}$. Then, replacing in $n^{T}$, we get $n^{T}=-\iota f_{x}^{T}(u-x)+f_{x}^{T}(v-y)=(-\iota(u-x)+(v-y)) f_{x}^{T}$ on $V\left(h_{i}, d_{1}, t\right)$. Since $d_{1}=-\iota(u-x)+(v-y)=0$ on $V\left(h_{i}, d_{1}, t\right)$, then $n^{T}=0$ on $V\left(h_{i}, d_{1}, t\right)$. We can prove in the same way that if $\left(f_{x}^{T}\right)-\iota\left(f_{y}^{T}\right) \in\left\langle h_{i}\right\rangle$ then $n^{T}=0$ on $V\left(h_{i}, d_{2}, t\right)$.

Reciprocally, let us suppose $n^{T}=0$ on $V\left(h_{i}, d_{1}, t\right)$ for some $i \in\{1,2, \ldots, s\}$. Since none of $n^{T}, h_{i}, d_{1}$ depend on $t, n^{T}=0$ on $V\left(h_{i}, d_{1}\right)$. Since $d_{1}=0$ on $V\left(h_{i}, d_{1}\right),\left\{\begin{array}{c}n^{T}=-f_{y}^{T}(u-x)+\iota f_{x}^{T}(u-x)=0 \\ n^{T}=\iota f_{y}^{T}(v-y)+f_{x}^{T}(v-y)=0\end{array}\right.$ on $V\left(h_{i}, d_{1}\right)$. Since $\left(-f_{y}^{T}+\iota f_{x}^{T}\right)=\iota\left(\iota f_{y}^{T}+f_{x}^{T}\right)$, we can rewrite the last system of equations as

$\left\{\begin{array}{c}\left(-f_{y}^{T}+\iota f_{x}^{T}\right)(u-x)=0 \\ \left(-f_{y}^{T}+\iota f_{x}^{T}\right)(v-y)=0\end{array}\right.$ on $V\left(h_{i}, d_{1}\right)$. The subvariety $V\left(h_{i}, d_{1}, u-x, v-y\right)=$ $V\left(h_{i}, u-x, v-y\right)$ is a one-dimensional variety in the two-dimensional variety $V\left(h_{i}, d_{1}\right)$. The set $V\left(h_{i}, d_{1}\right) \backslash V\left(h_{i}, d_{1}, u-x, v-y\right)$ is a dense open subset of $V\left(h_{i}, d_{1}\right)$. From the last system, we know that $\left(-f_{y}^{T}+\iota f_{x}^{T}\right)$ vanishes on this dense open subset. Now, we consider $V\left(h_{i}, d_{1},-f_{y}^{T}+\iota f_{x}^{T}\right)$. This is a closed projective set. We know that it contains the open set $V\left(h_{i}, d_{1}\right) \backslash V\left(h_{i}, d_{1}, u-x, v-y\right)$. Thus, it contains also its Zariski closure i.e. $V\left(h_{i}, d_{1}\right)$. Thus, $\left(-f_{y}^{T}+\iota f_{x}^{T}\right)$ vanishes on $V\left(h_{i}, d_{1}\right)$. Therefore, there exists $a, b \in k[x, y, u, v]$ such that $-f_{y}^{T}+\iota f_{x}^{T}=$ $a h_{i}+b d_{1}=a h_{i}+b(-\iota(u-x)+v-y)$. Let $a_{u v}$ be the sum of all the terms of $a$ containing the variables $u$ or $v$. Since $\left(-f_{y}^{T}+\iota f_{x}^{T}\right)$ does not depend on any of the variables $u$ and $v$, so $a_{u v} h_{i}=b(\iota u-v)$. Since the left hand side of this equality lies in $\left\langle h_{i}\right\rangle$, so does the right-hand side, hence $b \in\left\langle h_{i}\right\rangle$. Thus, $-f_{y}^{T}+\iota f_{x}^{T} \in\left\langle h_{i}\right\rangle$. Thus, $f_{x}^{T}+\iota f_{y}^{T} \in\left\langle h_{i}\right\rangle$. In the same way, we can prove that if $n^{T}=0$ on $V\left(h_{i}, d_{2}, t\right) \subset \mathbb{P}^{4}$ then $f_{x}^{T}-\iota f_{y}^{T} \in\left\langle h_{i}\right\rangle$.

We are going to analyse the dimension of the one-dimensional component of $W_{\infty}$. Let $l= \begin{cases}1 & \text { if } f_{x}^{T}+\iota f_{y}^{T} \in\left\langle h_{i}\right\rangle \text { or } f_{x}^{T}-\iota f_{y}^{T} \in\left\langle h_{i}\right\rangle \text { for some } i \in\{1,2, \ldots, s\} \text {. We } \\ 0 & \text { otherwise }\end{cases}$ will now introduce hypersurfaces, necessary in the proofs of the next lemma and of Theorem 3. If $X$ is a projective variety in $\mathbb{P}^{N}$ and $F \neq 0$ is a form on $X$ (i.e. a real-valued homogeneous polynomial function on $\mathrm{X}$ ), then we denote by $X_{F}$ the sub-variety of $X$, known as a hypersurface, defined by $F=0$.

Theorem 2. [9-Theorem 4, p. 57] If a form $F$ is not identically 0 on an irreducible projective variety $X$, then $\operatorname{dim} X_{F}=\operatorname{dim} X-1$.

In order to apply this theorem on the projective variety $W_{\infty}$ whose irreducibility is not known, we have determined if none of its irreducible components is contained in an irreducible component of $V(F)$. Indeed, if an irreducible component $V\left(h_{i}\right)$ is contained in an irreducible component $V\left(F_{i}\right)$ of $V(F)$, then $F_{i}$ vanishes on $V\left(h_{i}\right)$, and $\operatorname{dim} V\left(h_{i}\right) \bigcap V(F)=\operatorname{dim} V\left(h_{i}\right)$.

Lemma 5. The dimension of $W_{\infty}=V\left(f^{T}, n^{T}, d^{T}, t\right) \subset \mathbb{P}^{4}$ is equal to $l$. 
Proof. We start in $\mathbb{P}^{4}$, which has dimension 4. By Lemmas 2, 3] and repeated application of Theorem 2 (from $V\left(f^{T}\right)$ to $V\left(f^{T}, d^{T}\right)$, and from $V\left(f^{T}, d^{T}\right)$ to $\left.V\left(f^{T}, d^{T}, t\right)\right)$ we get that $V\left(f^{T}, d^{T}, t\right)$ has dimension $4-3=1$. Thus, the dimension of $W_{\infty}$ is 0 or 1 . By Theorem 2 the dimension of $W_{\infty}$ is 0 if, and only if, $l=0$ by Lemma 4 .

The following two lemmas give the degrees of two one-dimensional components, when these exist. The one-dimensional component at infinity and the one-dimensional component of $W_{S}$. They will allow us to conclude with the degree of the offset in Theorem 3. For this theorem, but also more generally, we recall the notion of degree. The degree of a $n$-dimensional projective variety $X \subset \mathbb{P}^{N}$ is the maximum number of points of intersection of $X$ with a projective linear subspace $\mathbb{P}^{N-n}$ in general position with respect to $X$ (see page 234 in [9]). Thus, the degree of a projective variety is the degree of its maximal dimensional component. We recall the definition of localisation at a prime ideal $\mathfrak{P} \subset k\left[t_{1}, \ldots, t_{m}\right]$, where $k$ is a field. We denote by $k\left[x_{1}, \ldots, x_{m}\right]_{\mathfrak{P}}$ the set of all rational functions $f / g$ such that $f, g \in k\left[x_{1}, \ldots, x_{m}\right]$ where $g \notin \mathfrak{P}$.

Definition 3. (adapted from $\sqrt{7}-D e f . A .8 .16, p .480])$ Let $F, G \in k[z, x, y]$ be homogeneous polynomials, let $p=\left(p_{0}: p_{1}: p_{2}\right) \in V(F) \cap V(G) \subset \mathbb{P}^{2}$, and let $\mathcal{M}_{p}=\left\langle p_{0} x-p_{1} z, p_{0} y-p_{2} z\right\rangle$ be the homogeneous ideal of $p$. Assume that $p_{0} \neq 0$ then, the intersection multiplicity of $V(F)$ and of $V(G)$ at $p$ is $\mu_{p}(F, G):=$ $\operatorname{dim}_{k}\left(k[z, x, y]_{\mathcal{M}_{p}} /\langle F, G\rangle\right)$.

Let $C_{\infty}$ be the component at infinity of the projective closure of $C$, i.e. $C_{\infty}=$ $V\left(f^{T}, t\right) \subset k^{2}$. Let $C_{S}$ be the affine subvariety of $C$ composed of all its singular points, i.e. $C_{S}=V\left(f_{x}, f_{y}, f\right) \subset k^{2}$.

Lemma 6. The one-dimensional component of $W_{\infty}$ has degree $2 l \sum_{p \in C_{\infty}} \mu_{p^{\prime}}\left(V\left(f^{T}\right), V\left(n^{T}\right)\right)$, where $p^{\prime}$ is an arbitrary point of $W_{\infty}$ whose projection on the projective $(t, x, y)$-plane is $p$.

Proof. The variety's degree is given by that of the component with maximum dimension. By lemma [5] $l=0$ iff $\operatorname{dim} W_{\infty}=0$ and we say that the degree of the (non-existant) 1-dimensional component is 0 . In the rest of the proof, we study the degree of the 1-dimensional component, assuming it exists, ie., for $l=1$. Note that this component may not be connected. There exist $i \in$ $\{1, \ldots, s\}, j \in\{1,2\}$ in the above notation, such that $n^{T}=0$ on $V\left(h_{i}, d_{j}, t\right)$, by Lemma 4. Hence, adding $n^{T}$ to $\left\langle d^{T}, f^{T}, t\right\rangle$ does not change its radical. Thus, the points of $W_{\infty}=V\left(f^{T}, d^{T}, n^{T}, t\right)$ and $V\left(\sqrt{\left\langle d^{T}, f^{T}, t\right\rangle}\right)$ are the same, but their multiplicities may differ. The one-dimensional component of $V\left(\sqrt{\left\langle d^{T}, f^{T}, t\right\rangle}\right)$ is constituted of all the points $p^{\prime}$ whose projection on the projective $(t, x, y)$-plane is a point $p$ of $C_{\infty} \subset \mathbb{P}^{2}$ and whose projection on the affine $(u, v)$-plane is the circle $V\left(d^{T}\right)$. The degree of the one-dimensional component of $V\left(\sqrt{\left\langle d^{T}, f^{T}, t\right\rangle}\right)$ equals the product of the degree of $d^{T}$ (which is 2) by the number of isolated points in $C_{\infty}$, counted with multiplicities. The reason is that each point of $C_{\infty}$ generates a one-dimensional component of $W_{\infty}$ with the $x, y$ coordinates of the 
point of $C_{\infty}$, and where the $t, u, v$ coordinates satisfy $d^{T}$. The degree of the onedimensional component of $W_{\infty}$ is twice the sum of the intersecting multiplicities of $V\left(f^{T}, n^{T}\right) \subset \mathbb{P}^{4}$ at the points $p^{\prime}$ for all the points $p$ of $C_{\infty}$.

Lemma 7. The degree of the one-dimensional component of $W_{S}$, defined at the beginning of this section, is: $2 \sum_{q \in C_{S}}\left(\mu_{q^{\prime}}(V(\bar{f}), V(\bar{n}))-1\right)$, where $q^{\prime}$ is an arbitrary point of $W_{S}$ whose projection on the affine $(x, y)$-plane is $q$. This component does not exist iff the degree vanishes.

Proof. Each point $q$ of $C_{S}$ induces a trivial equation $n$. Thus, at the level of $W_{S}$, each point $q$ of multiplicity $m$ induces a one-dimensional variety that consists of all the points $q^{\prime}$ whose projection on the affine $(x, y)$-plane is $q$ and whose projection on the projective $(t, u, v)$-plane is a projective circle centred at $q$ and of radius $R$ with multiplicity $m-1$ (the extraneous component may be simple). The only component at infinity of $W_{S}$ are the points $\left(0: x_{0}: y_{0}: 1: y_{0} \pm \iota\left(1-x_{0}\right)\right)$, where $\left(x_{0}, y_{0}\right)$ is a common root of $f^{T}, f_{x}^{T}(x, y)$, and $f_{y}^{T}(x, y)$. It follows that $W_{S}$ does not have a one-dimensional component at infinity. The points of $W_{S}$ are the same as the points of $V\left(\sqrt{\left\langle\overline{f_{x}}, \overline{f_{y}}, \bar{f}, \bar{d}\right\rangle}\right)$, but their multiplicities differ. The degree of the one-dimensional component of $V\left(\sqrt{\left\langle\overline{f_{x}}, \overline{f_{y}}, \bar{f}, \bar{d}\right\rangle}\right)$ equals the product of the degree of $\bar{d}$ (which is 2) by the number of isolated points in $C_{S}$. The degree of the one-dimensional component of $W_{S}$ is twice the sum of the multiplicities of $V(\bar{f}, \bar{n}) \subset \mathbb{P}^{4}$ at the points $q^{\prime}$ minus 1 for all the points $q$ of $C_{S}$.

Theorem 3. Let $p^{\prime}$ and $q^{\prime}$ be defined as in Lemmas 6 and 7 . The degree of the generalised $R$-offset $\mathcal{O}$ to an algebraic curve $V(f) \subset k^{2}$ of degree $m$, such as $f$ is square free is :

$$
2 m^{2}-2 l \sum_{p \in C_{\infty}} \mu_{p^{\prime}}\left(V\left(f^{T}\right), V\left(n^{T}\right)\right)-2 \sum_{q \in C_{S}}\left(\mu_{q^{\prime}}(V(\bar{f}), V(\bar{n}))-1\right) .
$$

Proof. Bézout's Theorem [9] states that, for projective varieties, the degree of the intersection of two varieties is generically equal to the product of the varieties' degrees. Therefore, the degree of $W$ is generically $2 \mathrm{~m}^{2}$. The quasi-projective varieties $W, W_{a}, W_{\infty}$, and $W_{S}$ are related by $W_{a}=W \backslash\left(W_{\infty} \cup W_{S}\right)$. Since $W$ is defined by three polynomials, its dimension is at least 1 according to Theorem 2. Since $n$ has two more variables than $f$ and the same degree, $n$ can not identically vanish on $V(\bar{f})$. Hence, $\operatorname{dim} V(\bar{f}, \bar{n}) \leq 2$, by Theorem 2 . Since $d$ has coefficients that are polynomials in $R, R \neq 0$ and the coefficients of $f$ and $d$ do not depend on $R, d$ can not identically vanish on $\bar{B} \cap \bar{N}$. Thus, the dimension of $W$ is exactly one by the same theorem. Since $W$ is one-dimensional, the degree of $W$ is the sum of the degrees of its one-dimensional components. Thus, the degree of $W_{a}$ equals the degree of $W$ minus the degree of the one-dimensional component of $W_{\infty} \cup W_{S}$. Since $W_{S}$ has no one-dimensional component at infinity (see proof of Lemma 7), the one-dimensional components of $W_{\infty}$ and of $W_{S}$ are 
disjoint, and the degree of the one-dimensional component of $W_{\infty} \cup W_{S}$ is the sum of the degrees of the one-dimensional components of $W_{\infty}$ and of $W_{S}$. By Lemmas [6] and [7, the degree of $W_{a}$ is given by Equation 1] By definition, $t$ never vanishes on $W_{a}$. Finally, $\mathcal{O}$ is the image of $W_{a}$ by the canonical projection $\pi: \mathbb{P}^{4} \backslash\{(1: 0: 0: 0: 0)\} \rightarrow k^{2}:(t: x: y: u: v) \mapsto\left(\frac{u}{t}, \frac{v}{t}\right)$ that is a one-to-one mapping. Indeed, there is generically only one point of the curve corresponding to a point of the generalised offset. Thus, the result.

By applying the results from this section, we get [1] the following degrees for conic offsets:

\begin{tabular}{|c|c|c|c|c|}
\hline Conic & ellipse/hyperbola & parabola & circle & two lines \\
\hline Offset degree & 8 & 6 & 4 & 4 \\
\hline
\end{tabular}

\section{The Generalised Offset to a Conic}

We will first review the main concepts and results about sparse resultants (see also [10, 3, 6]) that will be used hereafter. The resultant of $n+1$ polynomials $f_{1}, \ldots, f_{n+1}$ in $n+k$ affine variables is a polynomial in $k$ variables that characterises the solvability of the system of polynomial equations $f_{1}\left(x_{0}, \ldots, x_{n+k}\right)=$ $\cdots=f_{n+1}\left(x_{0}, \ldots, x_{n+k}\right)=0$. Thus, it allows the elimination of $n$ variables, and is therefore also called eliminant. The theory of sparse elimination has been widely covered in 10. The degree of the classical projective resultant in the coefficients of each polynomial is the Bézout number of the other $n$ polynomials (i.e., the product of their total degrees). Sparse resultants generalise the projective resultant and exploit the monomial structure of the polynomials expressed by the Newton polytope. The degree of the sparse resultant per polynomial, that is a sum of mixed volumes, is in general lower than that of the projective resultant.

The support $A_{i}$ of a polynomial $f_{i} \in k\left[x_{1}^{ \pm 1}, \ldots, x_{n}^{ \pm 1}\right]$ is the set of exponent vectors in $\mathbb{Z}^{n}$ corresponding to non-zero coefficients, i.e. $f_{i}=\sum_{a \in A_{i}} c_{a} x^{a}, c_{a} \neq 0$. The Newton polytope $Q_{i}$ of $f_{i}$ in $\mathbb{R}^{n}$ is the convex hull of $A_{i}$, i.e. the smallest convex set containing $A_{i}$. For example, for the strophoid of equation $y^{2}-x^{2}-$ $x^{3}=0$, the exponent vectors are: $(0,2),(2,0)$, and $(3,0)$. A polytope $Q \subset$ $\mathbb{R}^{n}$ has an $n$-dimensional volume, which will be denoted hereafter $\operatorname{Vol}(Q)$. The Minkowski sum $A+B$ of point sets $A$ and $B$ in $\mathbb{R}^{n}$ is the point set $A+B=$ $\{a+b \mid a \in A, b \in B\}$ (see example on Figure 21). Let $n$ polytopes $Q_{1}, \ldots, Q_{n} \subset$ $\mathbb{R}^{n}$ whose vertices belong to $\mathbb{Z}^{n}$. A polyhedral subdivision of a point set $S$ is a collection of polyhedra whose union equals $S$, such that each intersection of two polyhedra of the same dimension is another polyhedron in the subdivision of lower dimension. Let $Q=Q_{1}+\cdots+Q_{m} \subset \mathbb{R}^{n}$ be a Minkowski sum of polytopes, and assume that $Q$ has dimension $n$. Then a subdivision $R_{1}, \ldots, R_{s}$ of $Q$ is a mixed subdivision [4-Definition 6.5, page 344] if each cell $R_{i}$ can be written as a Minkowski sum $R_{i}=F_{1}+\cdots+F_{m}$ where each $F_{i}$ is a face of $Q_{i}$ and $n=\operatorname{dim}\left(F_{1}\right)+\cdots+\operatorname{dim}\left(F_{m}\right)$ (see example on Figure 2). The mixed volume is the sum of the volumes of the mixed cells [4-Theorem 6.7] (see example on 


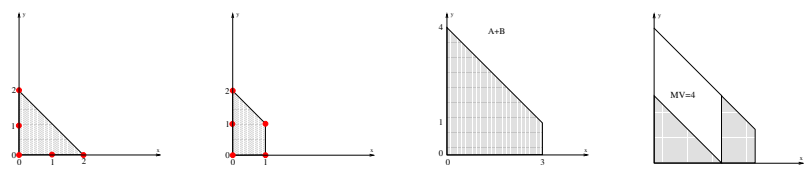

Fig. 2. The Minkowski sum (third diagram) of the point sets $A$ (first) and $B$ (second) and its mixed subdivision (filled-in cells are copies of Newton polytopes and mixed cells are unfilled)

Figure 2). If we replace a polytope $Q_{i}$ by a polytope $Q_{i}^{\prime}$ included in it, the mixed volume of the $Q_{i}$ does not increase.

A conic $C$ can be defined implicitly as the variety defined by a second degree formal polynomial: $f(x, y)=\alpha x^{2}+\beta x y+\gamma y^{2}+\delta x+\epsilon y+\zeta=0$. We shall compute an implicit equation of its generalised offset. The partial derivatives are $f_{x}=2 \alpha x+\beta y+\delta$ and $f_{y}=\beta x+2 \gamma y+\epsilon$. An equation of the normal $N=V(n) \subset k^{4}$ to the original conic at the point $(x, y)$ is: $n(x, y, u, v)=$ $-(\beta x+2 \gamma y+\epsilon)(u-x)+(2 \alpha x+\beta y+\delta)(v-y)=0$. If a conic is not degenerate (proper conic different from the union of two lines), then it has no singular points, and $S=\emptyset$. The generalised offset to a conic is the Zariski closure of the projection of the affine variety $\mathrm{V}(f, n, d) \backslash V\left(f_{x}, f_{y}\right)$ onto the $(u, v)$ plane. This is a subvariety of the Zariski closure of the projection of $V(f, n, d)$. Thus the equation of the generalised offset is a factor of the equation of the Zariski closure of the projection of $V(f, n, d)$, which is the resultant of $f, n, d$ expressing the elimination of $x, y$. The objective in the computations is to simplify the polynomials, while producing a system of equations equivalent to the original one. In the case of the sparse resultant computation, this has been achieved by replacing one polynomial by a linear combination of polynomials, and having a Newton polytope inscribed in the Newton polytope of the original polynomial. The sparse resultants have been computed thanks to the sparse resultant software developed by Emiris [6, 5]. We computed the determinant of this matrix and its factorisation. The degree of the offset to conics allowed us to identify the factor that corresponds to an implicit equation of the generalised offset. In the case where $\alpha$ and $\beta$ are different from 0 , we call the conic "generic". Hereafter, all conics are generic.

The polynomial $n$ can be rewritten in the following way: $n(x, y, u, v)=$ $\beta x^{2}-\beta y^{2}+2(\gamma-\alpha) x y+(-\beta u+\epsilon+2 \alpha v) x+(\beta v-\delta-2 \gamma u) y+(-\epsilon u+$ $\delta v)=0$. The monomial in $x^{2}$ can be eliminated if we replace $n(x, y, u, v)$ by $\alpha n(x, y, u, v)-\beta f(x, y)$ (see Figure 3 ). Similarly, if we replace $d(x, y, u, v)$ by $f(x, y)-\alpha d(x, y, u, v)$, the monomial in $x^{2}$ disappears (see Figure 3). The mixed volumes are $M V(f, \alpha n-\beta f)=M V(f, f-\alpha d)=4$ (see Figure 4), and $M V(\alpha n-\beta f, f-\alpha d)=3$. We get an equation for the generalised offset as the sparse resultant of $f, \alpha n-\beta f$ and $f-\alpha d$. Hereafter, all the implicit equations are publicly available at: http:// pages.cpsc.ucalgary.ca/antonf.

However, it is possible to further simplify the equation of a non-degenerate conic by using a coordinate system with origin at one of the foci (in the case of an ellipse or hyperbola) or the apex (in the case of a parabola), and one 

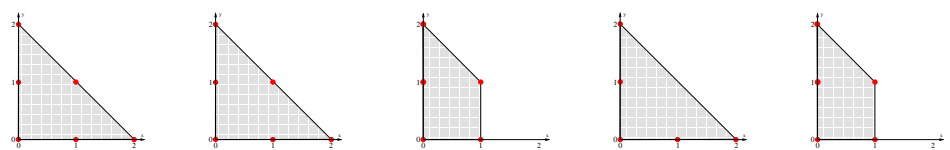

Fig. 3. The Newton polytopes of $f, n, \alpha n-\beta f, d$, and of $f-\alpha d$
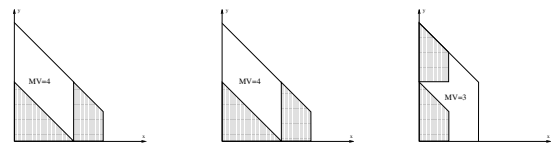

Fig. 4. The mixed volumes of $f$ and $\alpha n-\beta f$, of $\alpha n-\beta f$ and $f-\alpha d$, and of $f$ and $f-\alpha d$
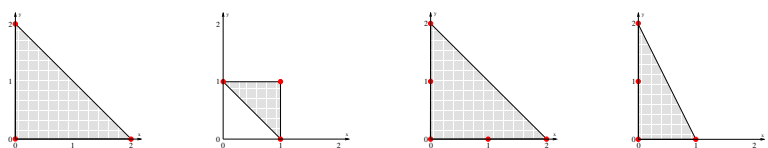

Fig. 5. The Newton polytopes of $f, n, d$ and of $a d-f$ for ellipses and hyperbolas
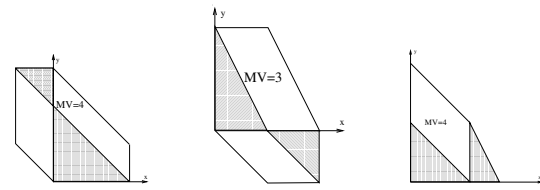

Fig. 6. A mixed subdivision and mixed volume computation of $f, n$, of $n, f-d$, and of $f, f-d$ for ellipses and hyperbolas

of the axes being the axis of the conic. By a simple change of coordinates, we can obtain easily the general equation of the generalised offset. Then, an ellipse or hyperbola simplifies to $\frac{x^{2}}{a^{2}} \pm \frac{y^{2}}{b^{2}}-1=0$, assuming $a b \neq 0$. We can get an equivalent equation $x^{2}+c y^{2}+e=0$, where $e \pm b^{2} c=0$. Let $f:=x^{2}+c y^{2}+e$. It is easy to see from Figure 5 that no linear combination of $f$ and $d$ has terms in common with $n$. If we replace $d(x, y, u, v)=(u-x)^{2}+(v-y)^{2}-R^{2}=0$ by $f(x, y)-d(x, y, u, v)$, the monomial in $x^{2}$ disappears (see Figure 5). The mixed volumes are $M V(f, n)=M V(f, f-d)=4$ and $M V(n, f-d)=3$ (see Figure 6). We get an equation for the generalised offset as the sparse resultant of $f, n, f-d$ in the variables $u$ and $v$. Examples are shown in Figure 7.

In the case of a parabola, the equation of the conic in a coordinate system with origin at the summit of the parabola, and one of the axes being the axis of the parabola, simplifies to $y^{2}-2 p x=0$. The polynomial defining $N=V(n) \subset k^{4}$ can be rewritten in the following way: $n(x, y, u, v)=-2 y u+2 x y-2 p v+2 p y=0$. It is easy to see from Figures 8 that no linear combination of $f$ and $d$ has terms 

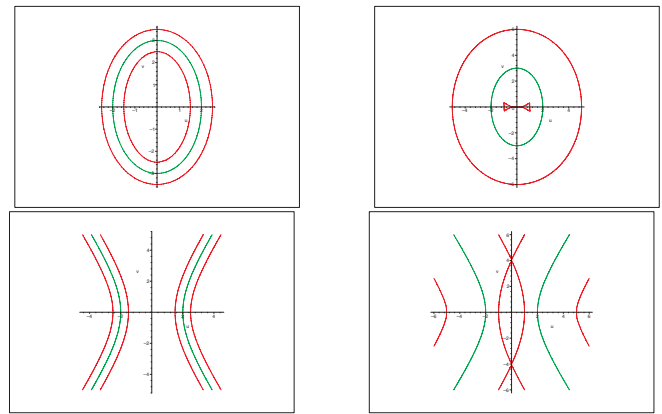

Fig. 7. Up: an ellipse with its 0.5-generalised offset (left) and its 3-generalised offset (right). Down: an hyperbola with its 0.5-generalised offset (left) and its 3-generalised offset (right)
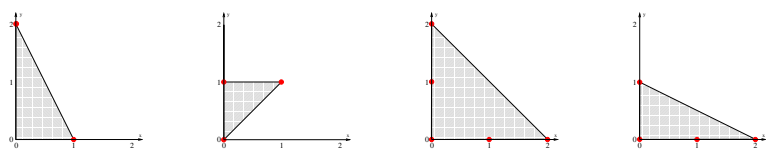

Fig. 8. The Newton Polytopes of $f, n, d$, and of $f-d$ for parabolas
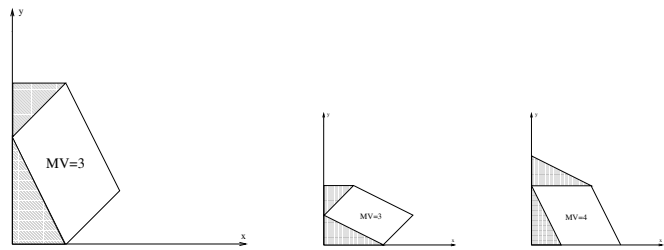

Fig. 9. The mixed volumes of $f, n$, of $n, f-d$ and of $f, f-d$ for parabolas
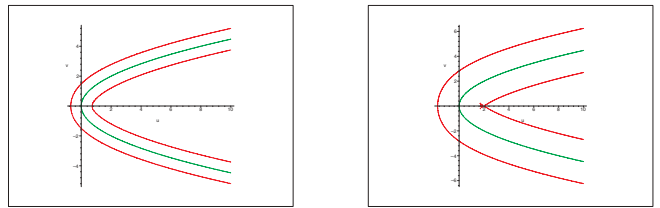

Fig. 10. A parabola and its 0.5-generalised offset. The same parabola and its 3-generalised offset

in common with $n$. If we replace $d(x, y, u, v)=(u-x)^{2}+(v-y)^{2}-R^{2}=0$ by $f(x, y)-d(x, y, u, v)$, the monomial in $y^{2}$ disappears (see Figure 8). The mixed volumes are $M V(f, n)=M V(n, f-d)=3$ and $M V(f, f-d)=4$ (see Figure 9). We get an equation for the generalised offset as the sparse resultant of $f, n, f-d$ in the variables $u$ and $v$. Examples are shown in Figure 10. 


\section{Conclusions}

We have obtained a general formula for the degree of the offset to an algebraic curve defined in its most general setting. We have obtained an implicit equation of the generalised offset to a conic defined by a formal polynomial, and simplified equations in the two cases of a circle, an ellipse or an hyperbola, and a parabola. This implicit equation of the generalised offset to a conic has been used in order to compute the Delaunay graph for conics and for semi-algebraic sets (see [1]).

\section{References}

1. F. Anton. Voronoi diagrams of semi-algebraic sets. PhD thesis, The University of British Columbia, Vancouver, British Columbia, Canada, January 2004.

2. E. Arrondo, J. Sendra, and J. R. Sendra. Genus formula for generalized offset curves. J. Pure Appl. Algebra, 136(3):199-209, 1999.

3. J. F. Canny and I. Z. Emiris. A subdivision-based algorithm for the sparse resultant. J. $A C M, 47(3): 417-451,2000$.

4. D. Cox, J. Little, and D. O'Shea. Using algebraic geometry. Springer-Verlag, New York, 1998.

5. I. Emiris. A general solver based on sparse resultants: Numerical issues and kinematic applications. Technical Report 3110, SAFIR, 1997.

6. I. Z. Emiris and J. F. Canny. Efficient incremental algorithms for the sparse resultant and the mixed volume. J. Symbolic Comput., 20(2):117-149, 1995.

7. G.-M. Greuel and G. Pfister. A Singular introduction to commutative algebra. Springer-Verlag, Berlin, 2002.

8. C. M. Hoffmann and P. J. Vermeer. Eliminating extraneous solutions in curve and surface operations. Internat. J. Comput. Geom. Appl., 1(1):47-66, 1991.

9. I. R. Shafarevich. Basic algebraic geometry. 1. Springer-Verlag, Berlin, second edition, 1994. Varieties in projective space, Translated from the 1988 Russian edition and with notes by Miles Reid.

10. B. Sturmfels. Sparse elimination theory. In Computational algebraic geometry and commutative algebra (Cortona, 1991), Sympos. Math., XXXIV, pages 264-298. Cambr. Univ. Press, Cambridge, 1993. 